\title{
Prognostic significance of radiological bone involvement in childhood acute lymphoblastic leukaemia
}

\author{
G. MASERA, V. CARNELLI, M. FERRARI, M. RECCHIA,* AND F. BELLINI
}

From the Paediatric Clinic and Department of Radiology, University of Milan, Italy

SUMMARY In 98 children with acute lymphoblastic leukaemia, aged 1 to 12 years, the prognostic significance of radiological bone involvement was studied. The mean duration of remission and of survival was much shorter in cases with multiple bone involvement ( 3 or more bones) than in those where bone involvement was absent. In those cases presenting with 1 or 2 bone lesions no statement of prognostic significance can be made at this stage. A radiological skeletal survey should be made in all children presenting with leukaemia to identify those (about $15 \%$ ) having multiple bone lesions and therefore a poor prognosis, in order that they can be given more intensive therapy.

The outlook for leukaemic patients where there is early bone involvement has been reported to be unfavourable in some series but not in others (Kundel et al., 1964; Nies et al., 1965; Aur et al., 1972). We have analysed a series of 98 children with acute lymphoblastic leukaemia (ALL), with a view to answering two questions. (1) Does leukaemic bone involvement correlate with the course of ALL? (2) Is there a syndrome in which diffuse skeletal lesions combined with characteristic haematological and clinical findings imply a poor prognosis?

\section{Materials and methods}

Ninety-eight children with ALL, between the ages of 1 and 12 years were followed between 1963 and 1975. Any one of the following radiological changes in bones were considered as leukaemic. (1) Metaphyseal bands of rarefaction; (2) osteoporosis and/or osteolytic lesions (destruction of bone tissue ranging from small, irregular, or punched-out areas to larger, Paget-like alterations in the bone structure); (3) periosteal reaction.

Cases were divided into three groups. Group 0: no evidence of bone lesions on $x$-ray (48 cases). Group I: radiological findings limited to one or two skeletal segments (34 cases). Group II: radiological lesions in more than two bone segments (16 cases).

\section{Received 13 December 1976}

*Present address: Biostatistic Unit, Mario Negri Institute of Pharmacological Research, Milan.
We also considered the following factors: age, sex, number of skeletal lesions, bone pain, WBC count, the interval between the first onset of symptoms and haematological diagnosis, response to therapy, median duration of complete remission, and median survival. Each factor was analysed statistically, using parametric (Linton and Gallo, 1975) and nonparametric (Ryan, 1959; Linton and Gallo, $1975)$ tests. The usual methodology for determining curves and standard errors (SE) of the median period of remission and survival times was used. All patients were treated similarly, regardless of the presence or absence of bone lesions. The treatment regimens used over this 12-year period may be summarized as follows.

1963-1965 prednisone, 6-mercaptopurine, and methotrexate (6MP-MTX); no prophylactic treatment to the CNS. 1965-1971 prednisone, vincristine (VCR), 6MP-MTX maintenance, VCR-vinblastine reinduction; prophylaxis for meningeal leukaemia with intrathecal MTX every 3 months. 1971-1973 L-asparaginase for 1 week, then prednisone-VCR, 6MP-MTX maintenance, VCR reinduction; prophylaxis of meningeal leukaemia with intrathecal MTX. 1973-1975 prednisone-VCR, 6MP-MTX maintenance, VCR reinduction; prophylaxis of meningeal leukaemia with intrathecal MTX and skull irradiation 2400 rads.

\section{Results (Table)}

Duration of complete remission (Fig. 1) for group II patients (7.4 months) was very short when com- 
Table Summary of data in 98 cases of $A L L$

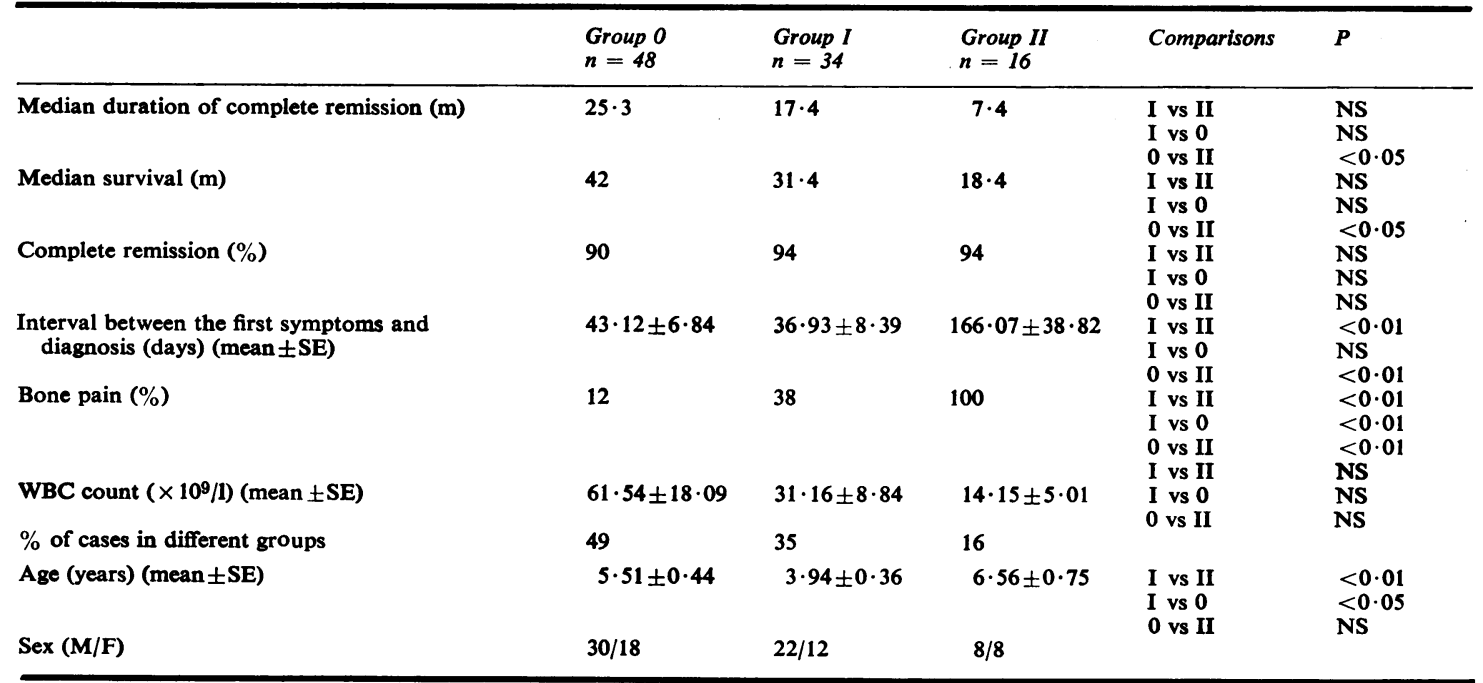

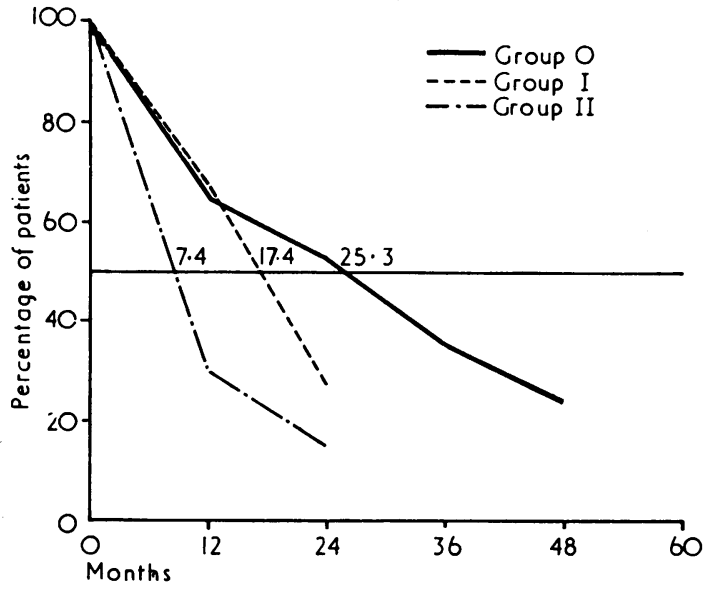

Fig. 1 Median duration of complete remission.

pared with group 0 (25.3 months). No significant difference was found between groups 0 and I (17.4 months) or between groups I and II. Median survival time (Fig. 2) also showed a significant difference when group II (18.4 months) was compared with group 0 (42 months). Remission induction rate: the percentage of patients who attained complete remission did not differ between the three groups. Interval between onset of symptoms (bone pain, fever, anaemia, haemorrhagic manifestations) and haematological diagnosis of ALL: for group II (166 days) this was longer $(P<0.01)$ than in groups 0 (43 days) or I (37 days) (Kruskal-Wallis statistical test and Ryan's procedure in combination). Incidence of bone pain: this was

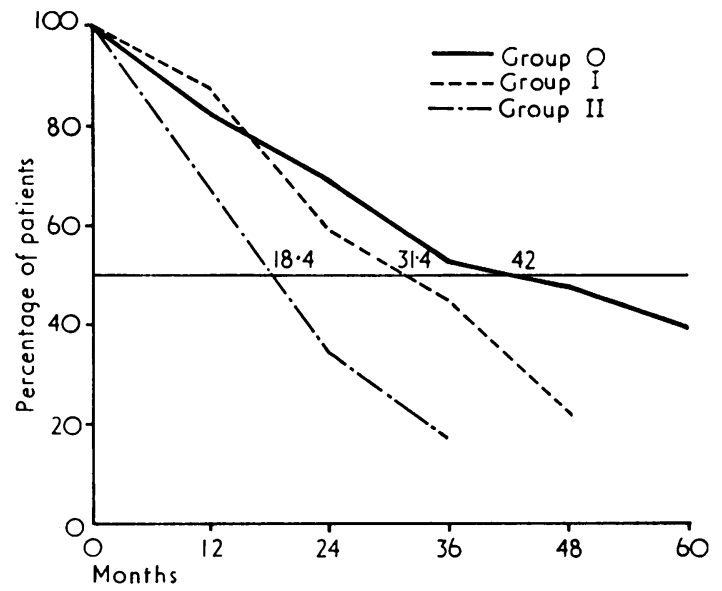

Fig. 2 Median survival.

highest in group II, lowest in group 0 , and intermediate in group I (Fisher's exact test). WBC count: though the median value was $61540 / \mathrm{mm}^{3}$ $\left(61 \cdot 54 \times 10^{9} / \mathrm{l}\right)$ in group $0,31160 / \mathrm{mm}^{3}\left(31 \cdot 16 \times 10^{9} / \mathrm{l}\right)$ in group $\mathrm{I}$, and $14150 / \mathrm{mm}^{3}\left(14 \cdot 15 \times 10^{9} / 1\right)$ in group II, there were no significant differences between the three groups. This was perhaps due to the use of a nonparametric test (Kruskal-Wallis test) which was necessary because of the variability of the data.

Frequency and type of skeletal lesions: in $\mathbf{5 0}$ of the patients the $x$-ray findings were characteristic of leukaemia. 34 of the 50 had lesions limited to 1 or 2 skeletal segments (group I), but the other $16 \mathrm{had}$ involvement of more than 2 segments. The fre- 
quency with which various bones were involved is shown in Fig. 3. Age: there was no significant difference (Duncan's new multiple range test), between groups $\theta$ (5.5 years) and II (6.6 years), but the differences between groups I ( 3.9 years) and II and between groups I and 0 were significant. Sex: there was no significant difference between the three groups (group 0, $63 \%$ males, group I, $65 \%$ and group II, $50 \%$ ).

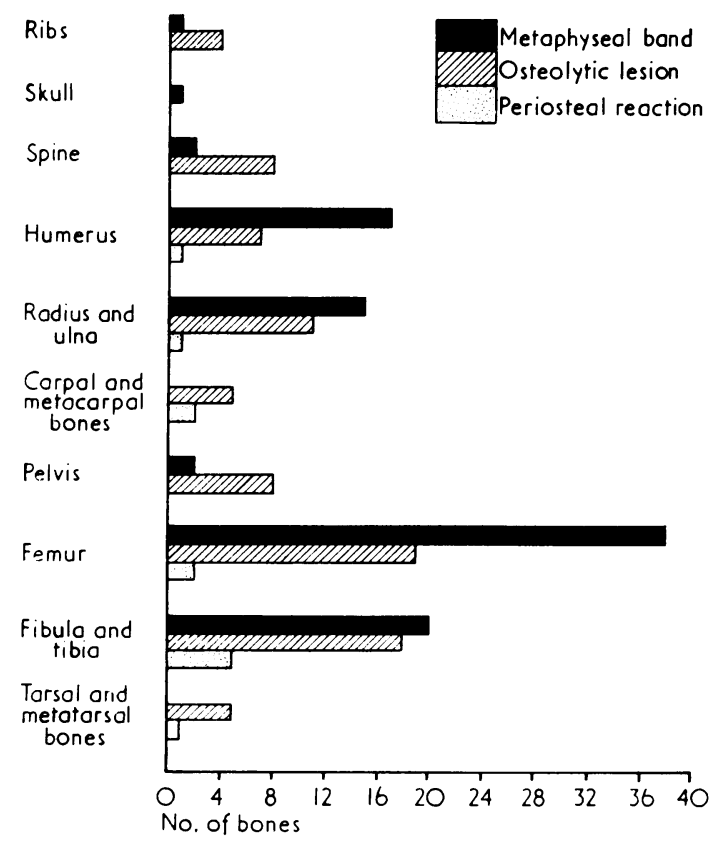

Fig. 3 Sites and types of bone lesions found in 98 children presenting with acute lymphocytic leukaemia.

\section{Discussion}

Selection of those ALL cases with an unfavourable prognosis, with a view to giving them intensive therapy without exposing the other patients to the risks of such treatment, is an aim which is generally recognized (Miller, 1975), but the possible prognostic significance of skeletal lesions has not been much studied. Kundel et al. (1964) drew attention to the fact that certain cases of leukaemia characterized by bone pain, bone necrosis, and leucopenia responded poorly to therapy. Nies et al. (1965) confirmed this in a large retrospective study of 316 patients examined post mortem, and they suggested that such cases might represent a particular clinicopathological complex. Aur et al. (1972), however, in a study of 221 children with ALL found no correlation between initial radiological bone involvement and prognosis, while Khanna et al. (1975), on the basis of 5 cases of ALL with diffuse skeletal lesions suggested that the presence of such lesions was associated with a short survival time. We have reconsidered the problem, comparing cases with multiple skeletal involvement (group II) with those having either no bone lesions (group 0) or lesions in only 1 or 2 skeletal segments (group I). One firm conclusion emerged, group II were found to have significantly shorter durations of both complete remission and survival when compared with group 0 .

Despite the marked differences between groups I and II in the duration of complete remission (group II, 7.4 months; group I, 17.4 months) and in median survival time (group II, 18.4 months; group I, 31.4 months), these differences failed to attain statistical significance, possibly due to the small number of cases. Again, when comparing group 0 with group I no statistically significant differences were shown. The prognostic significance of the presence of 1 or 2 bone lesions remains therefore undecided.

The possible association of other haematological and clinical findings with skeletal lesions, as suggested by Nies et al. (1965), was also studied. We confirmed that the form of childhood ALL which presents with extensive skeletal lesions with severe and constant pain, a long interval between the onset of first symptoms and haematological diagnosis, and leucopenia, carries an unfavourable outlook. $16 \%$ of our cases fell into this pattern, in agreement with the figure of $15 \%$ given by Kundel et al. (1964) and Nies et al. (1965). Thus about $15 \%$ of cases of childhood ALL will present with this recognizable clinicohaematological pattern carrying a predictably poor prognosis. It therefore seems important to make a complete radiological skeletal survey in every case of ALL in order to identify this group of cases which will merit more intensive therapy.

\section{References}

Aur, R. J. A., Westbrook, W., and Riggs, W., Jr. (1972). Childhood acute lymphocytic leukemia. Initial radiological bone involvement and prognosis. American Journal of Diseases of Children, 124, 653-654.

Khanna, K. K., Mazumdar, H., Ghosh, S. H., and Zingde, K. D. (1975). Skeletal changes in leukemia in children. Indian Pediatrics, 12, 430-433.

Kundel, D. W., Brecher, G., Bodey, G. P., and Brittin, G. M. (1964). Reticulin fibrosis and bone infarction in acute leukemia. Implications for prognosis. Blood, 23, 526-544.

Linton, M., and Gallo, P. S., Jr. (1975). The Practical Statistician. Brooks/Cole, Monterey, California. 
Miller, D. R. (1975). Prognostic factors in childhood leukemia. Journal of Pediatrics, 87, 672-675.

Nies, B. A., Kundel, D. W., Thomas, L. B., and Freireich, E. J. (1965). Leucopenia, bone pain and bone necrosis in patients with acute leukemia. A clinicopathologic complex. Annals of Internal Medicine, 62, 698-705.
Ryan, T. A. (1959). Multiple comparisons in psychological research. Psychological Bulletin, 56, 26-47.

Correspondence to Dr. G. Masera, Clinica Pediatrica G. e D. De Marchi, 20122 Milano, via Commenda 9, Italy. 